Chapter 2

\title{
Placenta Changes During Pregnancy with Thrombophilia - Influences of Low Molecular Weight Heparin Therapy
}

\author{
P. Ivanov and Tsv. Tsvyatkovska \\ Additional information is available at the end of the chapter \\ http://dx.doi.org/10.5772/56912
}

\section{Introduction}

When there is pregnancy establishment, three separate individuals are involved in the process: the fetus, the mother, and the father. Each has its own unique genetic material, and each has its own physiologic relationship to the others in the triad. In addition to the genetic heterogeneity, there is also physiologic complex bound between the mother and fetus concluded in double circulation of the placenta. Both maternal and fetal health affects the placenta circulation, and health is a combination of genetics and environment, which add further complexity to the situation.

A recent hypothesis has been given to evaluate lesions in the placenta that are or could be associated with hypoxia of either the fetus or mother part of placental circulation. The placenta is a two-part composite organ and is sustained by blood from both the mother and the fetus. Therefore, thrombophilia (inherited or acquired) in the mother, which might result in thrombosis of the spiral arterioles, could be hypothesized to have one effect on the placenta, whereas thrombophilia in the fetus would be expected to occlude the fetal vasculature with a different pattern of pathology.

\section{Maternal thrombophilia and placenta pathology findings}

It is known that thrombotic tendencies in the mother associated with acquired thrombophilia, such as the antiphospholipid syndrome, may result in placental pathologic findings of decreased placental weight, infarcts, increased numbers of syncytial knots, "accelerated villous 
maturation" and atherosis, identical to the findings associated with severe pregnancy induced hypertension [1, 2]. Because the presumed pathogenesis of the placental pathology is thrombosis of decidual arterioles, it is a logical assumption that similar changes may be seen in the presence of maternal inherited thrombophilia. The present studies are still in contradiction regarding whether the presence of inherited thrombophilia in the mother and/or fetus is associated with placental pathologic changes $[3,4,5]$. There are many variables among the investigation that attempt to determine whether there is an association between inherited mother thrombophilia and placental pathology. Studies differ in many respects, including whether the mutation status of the mother or fetus is assessed, the type of placental lesions evaluated, and the ascertainment of the subjects.

Pathologic findings which could be supposed as a result of placental ischemia are multitude but only a couple of them could be connected with disturbance due to the presence of factors leading to thrombosis development $[6,7,8]$.

Lesions hypothesized to reflect maternal thrombotic disease include placentas with weight small for gestational age $\left(<10^{\text {th }}\right.$ percentile), infarcts, and increased numbers of syncytial knots (Tenney-Parker change). The placenta's infarcts have been defined as localized area of coagulative necrosis in the placenta's parenchyma which is confirmed histologically. Increased syncytial knots, described as excessive number of villi with prominent syncytial knots; although most commonly seen with maternal vascular underperfusion, was also assessed not only in maternal but also in fetus thrombophilia.

One of the prominent investigations concerning the impact of maternal thrombophilia on placenta changes during pregnancy was performed by Rogers et al. [9]. They found statistically significant association of increased syncytial knotting and hypervascular villi with maternal FVL mutation suggesting that hypoxia of the placental vascular bed occurs more frequently in mothers with FVL mutation than those without. Rogers et al. found more than three times prevalence of syncytial knots after investigation of 105 placenta specimens from FVL positive mothers compared with 225 controls (respectively 13\% versus $4 \%$ pathological findings, $\mathrm{p}=0.004$ ). The investigation was performed over deliveries of health newborns after 35 week of gestation and was controlled for presence of placenta's pathology influence factors such as pregnancy related hypertension, preeclampsia, small-for-gestational-age infants. They also found increased number of hypervascular villi in FVL positive mothers compared with controls $(10 \%$ versus $3 \%, \mathrm{p}=0.018)$. Placentas from infants heterozygous for FVL mutation had more avascular villi than controls (OR 2.9, 95\% CI 1.5-5.6, $\mathrm{p}=0.001$ ). This study was performed with statistical power to patients' follow up in a prospective manner and in addition, a single pathologist, blinded to the clinical data, reviewed the slides to maintain consistency of observation.

Gogia and Machin [10] found other placenta pathology connected with thrombophilia. They established that maternal floor infarction (MFI), massive perivillous fibrin deposition (MPVFD), and fetal thrombotic vasculopathy (FTV) are specific placental lesions with associations to recurrent adverse fetal outcomes and with maternal thrombophilia. Maternal floor infarction was defined as rind of fibrinoid lining the maternal surface of the placenta. Pervillous fibrin deposition was diagnosed as transmural and not involving the placental periphery 
only. Fetal arterial vasculopathy was confirmed when there was no evidence of cord blood flow restriction, and thrombi were identified in stem villous arteries, with downstream avascular villi floating in a patent intervillous space. In the total of 138 investigated placentas $77 \%$ of the identified thrombophilia were genetic, and $23 \%$ were acquired. Thrombophilia was identified in $40 \%, 23 \%$, and $71 \%$ respectively in cases of maternal floor infarction, perivillous fibrin deposition, and fetal thrombotic vasculopathy. The most common genetic thrombophilia was protein S deficiency, found in 39\% of cases followed by FVL established in 31\% of cases.

Four other published series investigated maternal and fetal thrombophilia in the context of specific placental lesions, including fetal thrombotic vasculopathy, maternal floor infarction, and massive perivillous fibrin deposition. Three of these showed an association between maternal thrombophilia and placental lesions [11, 12, 13]. Ariel et al [14] found no evidence of increased prevalence of FVL, FII 20210 G>A, and MTHFR 677 C > T in fetal DNA of 19 cases with a variety of placental lesions, including occlusive large fetal vessel disease, hyalinized avascular villi, and chorangiosis. However they did not include maternal floor infarction or perivillous fibrin deposition, and the investigation was limited to the three types genetic thrombophilia.

\subsection{Conclusion}

In summary, there are the alterations of the placenta indicative of the presence of inherited thrombophilia in the mother, although there are papers which have come to the conclusion that there is no clear relationship between thrombophilic mutations with placental abnormalities and/or adverse pregnancy outcomes. This is also confounded by the fact that the thrombophilic mutations are not equally frequent among the races and many studies had small sample sizes.

\section{Fetal thrombophilia and placenta pathology findings}

Hereditary and acquired coagulation abnormalities may contribute to hypercoagulability, especially during the second and third trimester of pregnancy which places the placenta and ultimately the fetus at risk for complications. Because the placenta is an organ with two separated by specific membrane circulations, fetal in the villi and maternal between the villi, placental thrombotic complications may include those contributed by either the mother or fetus.

Placental findings that indicate maternal thrombotic or thromboembolic events include placental infarcts in which the villi are infarcted because of maternal vascular compromise. In these cases the pathology findings are necrotic villi with collapsed or empty intervillous space (maternal lakes). Fairly rarer are placental findings that suggest a thrombosis in the fetal part of placental circulation. Fetal-side thrombosis (FST) is uncommon, but could be catastrophic for fetus fate because of the related high perinatal morbidity and mortality. Evidence of FST has been documented in cases of fetus congenital stroke $[15,16]$, renal vein thrombosis $[17$, $18]$, and by placental lesions $[19,20]$. Thereby the term FST is related not only with placental- 
fetal-vascular thrombosis, but also with fetal-visceral-vascular lesions. Placenta-related FST are documented by the occurrence of a couple of different histopathological findings. First, this is macroscopic or microscopic presence of thrombosis in the fetal circulation including villous vessels, chorionic plate vessels, umbilical vessels. Second, there is hemorrhagic endovasculitis (extravasated fetal RBCs) in livebirths, related with the presence of areas of occluded villous vessel with patent (open) intervillous space in the absence of any inflammatory signs of villitis $[21,22,23]$. The mentioned thrombosis in chorionic villi, chorionic plate or umbilical vessel has been reported in $4 \%$ to $5 \%$ of all placentas examined by histology [20]. So the relation between clinical appearance and pathology finding is frequently obscure. Additionally, some maternal disorders like diabetes mellitus, and placental abnormalities such as cord anomalies, velamentous vessels, chorioamnionitis with vascular inflammation ("funisitis" or chorionic plate "vasculitis"), and multigestation are associated with placental FST. However, these associations are exceptional and the above mentioned pregnancy complications have been related mainly with maternal side placental thrombosis [24, 25].

Frequently, most placental FST present in placentas with no other findings and in patients (mothers and infants) with no known risk factors. The clinical significance of placental FST is controversial. Some authorities regarding even a single thrombus is significant [19], whereas others attach no significance to a small isolated lesion [25]. The possible association of placental FST with severe fetal conditions such as cerebral palsy or fetal/infant visceral thrombosis [26] probably warrants clinical vigilance, but in the cases of absence of specific causes targeted follow-up is difficult.

One potential cause of placental FST are hereditary haemostatic aberrations. This finding suggests that the placenta may be the cause of placental vascular insufficiencies either from maternal or fetal side complications, as suggested by some authors [27, 28]. Recently, perinatal morbidity has been studied in relation to placental FST and the potential contribution of inherited hypercoagulability in the fetus is noted as an important differential diagnosis [13]. In these cases an examination of fetus carrier status for thrombophilic mutation has been considered.

A short prospective and extended retrospective investigation connected with fetal thrombophilia and fetal-side placental thrombosis has been performed by Vern et al [12]. They evaluated 148 placentas for FST and along with this a carrier status of the fetuses for FVL and FII $20210 \mathrm{G}>\mathrm{A}$ have been performed. FST was found only in 3\% of investigated placentas. One heterozygous fetus for FVL and another for FII $20210 \mathrm{G}>\mathrm{A}$ were found giving very low mutation incidences (less than $1 \%$, in disagreement with reported 3 to $5 \%$ appearance in health Caucasian population). Because of these results, the authors proceeded to analyze a larger number of placental FST in a separate retrospective study: Five of 27 study cases $(18.5 \%)$ of placental FST were identified as FVL heterozygotes by this assay. One case of heterozygosity for FVL was found among 21 control placenta cases without FTS. The numbers were small, however, compared with both sets of controls, the number of FVL in infants with placental FST was significantly increased $(\mathrm{P}<0.01)$. None of the known risk factors for placental FST were present in the FVL heterozygous cases: none had acute chorioamnionitis, maternal diabetes mellitus, suffered a cord accident, nor had membranous insertions of the cord. This 
excluded confounding factors which could be related with FST pathology. The apparent increase in the incidence of FVL in placentas with placental FST suggests that in some cases the cause of placental FST may be fetal thrombophilia. This is supported by the fact that in these identified FVL heterozygotes, none had any other known risk factor for developing placental FST. It should be noted that the mentioned study included only fetuses and placentas from normal pregnancies ended with live born fetus after 37 week of gestation. The histopathology of placenta of FVL positive stillbirth has not been discussed in this study. Because individuals with FVL carry a distinct increased risk for thrombotic complications, the authors suggest that infants (and potentially the parents of these infants), born of pregnancies complicated by FST in the placenta, should screened for FVL.

Ariel et al [14] investigated the histology findings in positive and negative FVL fetuses which were born after pregnancy with placental abruption, intrauterine growth restriction or preeclampsia. They have not found association between FVL and placental thrombotic changes, nevertheless there were 19 from 64 newborn with FVL thrombophilia (29.7\%).

Other authors [29] investigated carrier status for FVL in abortive materials of pregnancies ended in first and second trimester. Along with mutation establishment, the authors examined placentas for presence of infarcts on fetal placenta side. The placental infarctions on fetal side were defined as histology findings of occluded fetal stem artery associated with infarction of the terminal villi in the distribution of the occluded vessel. So, the maternal side infarction connected with heavy deposition of fibrin in the decidua beneath the placenta rather than arterial occlusion and ischemic necrosis of the villi have been excluded. The authors found greater than twice the carrier frequency of FVL mutation in spontaneously aborted fetuses $(8.6 \%)$ than in the control group $(4.2 \%), p=0.046$. Placentas with more than $10 \%$ surface occupied by fetal side infarction were found in $42 \%$ of FVL positive fetuses compared with only $1.9 \%$ in placentas from non FVL fetuses $(\mathrm{p}<0.0001)$. The results suggest that FVL mutation predisposes to spontaneous miscarriage and placental infarction in cases of fetal thrombophilia.

Von Kries at al. [30] perform study over 375 Caucasian children born after 37 week of gestation pregnancy with birth weight "in the lowest quartile" for respective week of gestation. They investigated children carrier status for FVL, FII 20210 G>A, protein C, S, antithrombin III deficiency. The authors found non-significant correlation (OR 1.53 95\% CI 0.76-3.08) between single mutation carrier status and significant correlation (OR 4.01 95\% CI 1.48-10.84) between two or more mutation carrier status and intrauterine grow retardation (IUGR). Proportion of IUGR among children with one and two mutations was respectively $27.3 \%$ and $40 \%$. So the authors concluded that fetal thrombophilia could be an additional cause of low birth weight.

Although it is difficult to gauge whether the presence of FVL or FII 20210 G>A may affect coagulation in the fetus, especially because fetal and neonatal levels of coagulation proteins are low and clotting indices such as prothrombin time are prolonged in comparison with adults [31], the presence of FST in placentas from pregnancies without other thrombotic risk factors strongly suggests that fetal hypercoagulopathy may exist. Concerning future treatment of FST or arranging actions against repeated incidences of FST in further pregnancy not very much 
could be done. As it has been known to date, the low molecular height heparins did not cross placenta so the predictable anticoagulant effect in fetus coagulation could not expected.

\section{Doppler ultrasound markers and thrombophilia presence. Influence of antithrombotic therapy on uterine and placental blood flow}

\subsection{Introduction}

The association between pregnancy complications and high incidence of acquired and congenital thrombophilia may indicate that disturbances in hemostasis lead to a prothrombotic state and may predispose affected individuals to either poor embryonic implantation in the endometrium or, later in pregnancy, to decreased placental perfusion. The proposed underlying mechanisms [32] include interference with trophoblast differentiation, inadequate placentation, or thrombosis of the placental vasculature, with consequent reduced placental perfusion, oxidative stress, and maternal endothelial dysfunction that is believed to trigger the hallmark biological and clinical manifestations of preeclampsia, IUGR or pregnancy loss.

Acquired thrombophilia such as antiphospholipid antibody syndrome has been shown to be associated with adverse pregnancy outcomes. Direct damage of trophoblast cells and uteroplacental thrombosis appear to lead to the fetal manifestations of poor growth, oligohydramnios and abnormal Doppler velocimetry. There is some evidence in antiphospholipid antibody syndrome that treatment with heparin corrects the abnormality in trophoblast invasion and thrombosis, and also decreases the fetal loss rate. Numerous conflicting studies suggest a relationship between adverse pregnancy outcomes and genetic thrombophilia. The pathogenesis of the disease is likely to be very similar to antiphospholipid antibody syndrome though data is limited. But there are considerable controversies about the influence of genetic thrombophilia on the ultrasound markers associated with blood flow in the uterine arteries, circulation in placental vessels and umbilical cord.

Failure of trophoblastic invasion has been found to be associated with uteroplacental insufficiency. Doppler measured parameters both in first and second trimesters have demonstrated an association between the increased impedance of flow in the uterine arteries and subsequent development of preeclampsia, IUGR, and fetal death. Doppler ultrasound predicts the development of severe preeclampsia with higher sensitivity and specificity, compared with pregnancy-induced hypertension. For example Papageorghiou et al [33] demonstrated that increased pulsatility index (PI) on Doppler ultrasound was identified in $41 \%$ of women who later developed preeclampsia. The sensitivities for preeclampsia requiring delivery before 36 , 34 , and 32 weeks were $70 \%, 81 \%$, and $90 \%$, respectively. Doppler ultrasound has also been used in the first trimester to predict preeclampsia. The likelihood ratio (LR) for the development of PE was about 5 and for those with normal Doppler results the LR was about 0.5 [34]. Similarly, the LR of developing IUGR is about 4 in women with evidence of increased impedance of blood flow on Doppler. The main preventive approach in this approach to escape this pregnancy complication has been connected with low molecular weight heparin applica- 
tion. LMWHs with long half-lives, resulting in the need for less frequent injections, have made them attractive for practical use during the 9 months of pregnancy. In addition, the widespread use over the last 10 years has shown that LMWHs are safer than unfractionated heparin (UH) during pregnancy. Many studies have indicated that LMWHs are one of the factors regulating trophoblast invasion, although the results were not always consistent. In 2006, Erden et al. [35] found the underlying mechanism involved in the improvement of trophoblast invasion using LMWH in patients with a history of miscarriage. They reported that enoxaparin can reduce Ecadherin expression but not laminin expression in rat pregnancy, which might modulate trophoblast invasion. The antithrombotic action and the amelioration of blood flow rheology of LMWH in developed intervillous space in second trimester of pregnancy are believed to improve Doppler sonographic markers. The discovery of discreet Doppler alterations in placental and uterine artery blood flow could help in early steps of pregnancy rescue.

\subsection{Doppler ultrasound markers and thrombophilia presence}

One of the first authors who investigated connection between thrombophilia and impaired blood flow during pregnancy were Grandone et al. [36] They found persistent in second trimester of pregnancy bilateral uterine artery notches during Doppler blood flow examination in 41 women with inherited thrombophilia (FVL, FII 20210 G>A, antithombin III, protein C or protein $S$ deficiency). This finding is associated with six-fold higher risk of having an adverse outcome compared with women without these thrombophilic conditions.

Other studies again evaluated the presence of an association between common prothrombotic factors and increased blood flow resistance in the fetomaternal circulation, connected with obstetric complications occurrence. The investigators [37] did not find an association between thrombophilia and blood flow in the fetomaternal circulation in nulliparous women.

Useful information was found in Doppler sound characteristics investigation of uterine artery flow in non-pregnant women with recurrent pregnancy loss history. Lazzarine et al [38] investigated PI values of uterine artery in midluteal phase of 230 women who had experienced two or more first trimester pregnancy loss (RPL). Uterine arteries PI values in RPL patients $(2.42 \pm 0.79)$ were significantly higher with respect to those found in the control group $(2.08 \pm$ $0.47)$. When patients were grouped according to the different RSA causes, the highest PI values were found among patients with uterine abnormalities $(2.82 \pm 1.0)$, antiphospholipid antibodies syndrome $(2.70 \pm 1.1)$, and unexplained RSA $(2.60 \pm 0.7)$. These values were significantly higher with respect to that found in the control group. No differences were observed in PI values between fertile patients and those with RSA due to thyroid abnormalities $(2.10 \pm 0.55)$, inherited thrombophilia $(2.03 \pm 0.45)$, autoimmune pathology $(2.34 \pm 1.18)$, and genetic anomalies $(2.47 \pm 0.54)$. Similar results were observed when patients were grouped according to primary and secondary RPL. According to the results, the increased resistance of uterine blood flow in non-pregnant uterus may be an important sign to some causes of RPL and may represent an independent indication of careful further pregnancy follow up.

When talking about fetus thrombophilic status, there should be mentioned the important influence of non-mother thrombophilic conditions and the disturbance of umbilical blood flow. Lindqvist et al [39] investigated umbilical artery Doppler velocimetry on 54 women in 
late pregnancy. They found that abnormal umbilical artery Doppler velocimetry was associated with an approximately seven-fold increased risk of fetoplacental thrombotic vasculopathy (OR: 7.5, 95\% CI: 1.3-44.3), ischemic lesions (OR: 7.5, 95\% CI: 1.2-46.1) and fetal carriership of FVL (OR: 8.2, 95\% CI: 1.5-43.5). The study gives power of FVL-fetus carriership and pregnancy complications connected with fetal demise.

\subsection{Influence of antithrombotic therapy on uterine and placental blood flow}

In the follow up of women with inherited and acquired thrombophilia using LMWH Cok et al [40] did not find significant positive influence of the therapy with heparins on the uterine artery blood flow. They concluded that the administration of LMWH (enoxaparine $40 \mathrm{mg}$ daily) throughout the pregnancy in patients with thrombophilia doesnot prevent theincreaseofuterine artery Doppler flow indices and IUGR, which is probably as a result of defective trophoblastic invasion. Investigation of Doppler parameters was performed during the 18-22-week period of gestation over 64 pregnant women who experienced minimum three previous pregnancy loss after ten weeks of gestation and who had acquired or inherited thrombophilia (positive lupus anticoagulant, antiphospholipid antibody, FVL, FII 20210 G>A, antithombin III, protein C or S deficiency). The authors found increased impedance to blood flow in the uterine arteries which is associated with increased risk for subsequent development of preeclampsia, IUGR, and perinatal death in thrombophilia presence. Despite LMWH therapy, the mean PI $(1.07 \pm 0.46$ for LMWH group and $0.91 \pm 0.31$ for control, $\mathrm{p}=0.036)$ and the mean RI $(0.59 \pm 0.12$ for LMWH group and $0.54 \pm 0.10$ for control, $p=0.021$ ) were significantly higher in the trombophilia group. These results should be interpreted with care because of relatively small investigated group and the diversity type of thrombophilia among pregnant women. Another open question is the dose regiment of LMWH therapy arranged accordingly thrombophilia type.

On thecontrary, Magriplesetal[41] found positiveeffect of anticoagulation therapy overDoppler sonographic finding in retrospective study of 51 women with inherited thrombophilia (FVL, FII 20210 G > A, MTHFR 677 C > T). In the treatment group thrombophilic women used unfractionated heparin or low molecular weight heparin for FVL and FII $20210 \mathrm{G}>\mathrm{A}$ in prophylactic doses. Heparin was used at prophylactic doses until the third trimester. In the third trimester, patients were advanced to therapeutic doses of heparin and switched to unfractionated heparin at 36 weeks. From the total of 178 monitored pregnancies, the authors reported abnormal ultrasounds significantly greater in the untreated compared with the treated with heparins pregnancies $(52.8 \%$ versus $27.9 \%, \mathrm{p}=0.024)$. Growth restrictions were more common in untreated pregnancies. There was a significantly decreased risk of oligohydramnios with treatment $(27.3 \%$ versus $7 \%$, $\mathrm{p}=0.03)$. Overall outcomes were significantly improved with the use of anticoagulation $(p<0.0001)$. In a part of the cases despite treatment, oligohydramnios and growth disturbances still occurred. As heparin does not cross the fetal side of the placenta, this may account for the persistence of the poor outcomes because of positive fetus thrombophilia phenotype. The study markedly established the connection between ultrasound parameters finding for growth, fluid and feto-placental blood flow in patients with pregnant thrombophilic women and the application of anticoagulant therapy.

In a small observational study Alkazaleh et al [42] also found beneficial effect of LMWH therapy over Doppler placental flow and fetal outcome in women with previous history with 
pregnancy complications. They also draw attention over earlier application of LMWH therapy in indicated cases (not later than 18 to 20 week of gestation). This opens the question for window of Doppler sonographic screening and well-timed therapeutic intervention in high risk pregnancy.

\subsection{Conclusion}

Giving the cost and potential side effects of heparins during pregnancy, the deployment of a strategy of placental function screening using Doppler ultrasound and adding antithrombotic therapy only in the accurate cases is an appropriate clinical strategy. LMWH's therapy should be reserved for high risk populations with inherited thrombophilia mutations (double mutation carriers or thromboembolic incidences history). In non-pregnant women the Doppler examination of the uterine artery represents a useful tool for screening women with a history of RPL and, therefore, should be included in the RPL diagnostic flow chart. This test provides the opportunity to identify women in whom appropriate therapeutic protocols may effectively improve the possibility for a successfully pregnancy.

\section{General conclusions}

The authors who found correlation between placenta pathological findings and thrombophilia strongly advocate thrombophilia testing in all cases in which the placenta shows signs of chronic ischemic disturbance. They also recommend a full-pedigree work up including fetal and father thrombophilia screening. This approach is coming from possible multigenic effect over placenta structure coming from both maternal and fetal genotype.

The results as a whole suggest that these unusual forms of fibrin deposition (not thrombosis), increased numbers of syncytial knots and maternal side placental infarcts are frequently associated with thrombophilia. In a part of cases - in maternal floor infarction there is $50 \%$ recurrence rate suggests an interaction between maternal and fetal thrombophilia, resulting in a "plane of fibrin deposition" in which the maternal and fetal circulations overlap across the entire placental bed. Only about in $40 \%$ of cases of placentas with histopathology showing chronic ischemia have identifiable thrombophilia. The reasons for this may include other thrombophilia not discovered or not investigated in the give study or presences of other (mechanical?) factors impede blood flow in the intervillous space.

\section{Author details}

P. Ivanov ${ }^{1,2}$ and Tsv. Tsvyatkovska ${ }^{2}$

1 Clinical Institute for Reproductive Medicine, Pleven, Bulgaria

2 Department of Biochemistry, Medical University of Pleven, Bulgaria 


\section{References}

[1] Levy, R. A, Avvad, E, Oliveira, J, \& Porto, L. C. Placental pathology in antiphospholipid syndrome. Lupus. (1998). SS85., 81.

[2] Abramowsky, C. R, Vegas, M. E, Swinehart, G, \& Gyves, M. T. Decidual vasculopathy of the placenta in lupus erythematosus. NEJM. (1980). , 303, 668-672.

[3] Redline, R. W. Thrombophilia and placental pathology. Clin Obstet Gynecol. (2006). , $49,885-894$.

[4] Katz, V. L. Di Tomasso J, Farmer R, Carpenter M. Activated protein C resistance associated with maternal floor infarction treated with low-molecular-weight heparin. Am J Perinatol. (2002). , 19, 273-277.

[5] Many, A, Schreiber, L, Rosner, S, Lessing, J. B, Eldor, A, \& Kupferminc, M. J. Pathologic features of the placenta in women with severe pregnancy complications and thrombophilia. Obstet Gynecol. (2001). , 98, 1041-1044.

[6] Kraus, F. T, Redline, R. W, Gersell, D. J, Nelson, D. M, \& Dicke, J. M. editors. of the Atlas of Nontumor Pathology. 2. Armed Forces Institute of Pathology; Washington, DC: American Registry of Pathology; (2004). Placental Pathology., 3

[7] Redline, R. W, Boyd, T, Campbell, V, Hyde, S, Kaplan, C, Khong, T. Y, Prashner, H. R, \& Waters, B. L. Maternal vascular underperfusion: nosology and reproducibility of placental reaction patterns. Pediatr Dev Pathol. (2004). , 7, 237-249.

[8] Redline, R. W, Ariel, I, Baergen, R. N, Desa, D. J, Kraus, F. T, Roberts, D. J, \& Sander, C. M. Fetal vascular obstructive lesions: nosology and reproducibility of placental reaction patterns. Pediatr Dev Pathol. (2004). , 7, 443-452.

[9] Rogers, B. B, Momirova, V, Dizon-townson, D, Wenstrom, K, Samuels, P, Sibai, B, Spong, C, Caritis, S. N, Sorokin, Y, Miodovnik, M, Sullivan, O, Conway, M. J, \& Wapner, D. RJ. Avascular villi, increased syncytial knots, and hypervascular villi are associated with pregnancies complicated by factor V Leiden mutation. Pediatr Dev Pathol. (2010). , 13, 341-7.

[10] Gogia, N, \& Machin, G. A. Maternal thrombophilias are associated with specific placental lesions. Pediatr Dev Pathol. (2008). , 11, 424-9.

[11] Arias, F, Romero, R, Joist, H, \& Kraus, F. T. Thrombophilia: a mechanism of disease in women with adverse pregnancy outcome and thrombotic lesions in the placenta. J Matern-Fetal Med (1998). , 7, 277-286.

[12] Vern, T. Z, Alles, A. J, Kowal-vern, A, Longtine, J, \& Roberts, D. J. Frequency of Factor V Leiden and prothrombin G20210A in placentas and their relationship with placental lesions. Hum Pathol (2000). , 31, 1036-1043. 
[13] Kraus, F, \& Acheen, V. I. Fetal thrombotic vasculopathy in the placenta: cerebral thrombi and infarcts, coagulopathies, and cerebral palsy. Hum Pathol (1999). , 30, 759-769.

[14] Ariel, I, Anteby, E, Hamani, Y, \& Redline, R. W. Placental pathology in fetal thrombophilia. Hum Pathol (2004). , 35, 729-733.

[15] Silver, R. K. MacGregor SN, Pasternak JF, et al: Fetal stroke associated with elevated maternal anticardiolipin antibodies. Obstet Gynecol (1992). , 80, 497-499.

[16] Koelfen, W, \& Freund, M. Varnholt V: Neonatal stroke involving the middle cerebral artery in term infants: Clinical presentation, EEG and imaging studies, and outcome. Dev Med Child Neurol (1995). , 37, 204-212.

[17] Oppenheimer, E. H. Esterly JR: Thrombosis in a newborn: comparison between infants of diabetic and nondiabetic mothers. J Pediatr (1965). , 67, 549-556.

[18] Alexander, F. Campbell WAB: Congenital nephrotic syndrome and renal vein thrombosis in infancy. J Clin Pathol (1971). , 24, 27-40.

[19] Kraus FT: Placental thrombi and related problemsSemin Diagn Path (1993). , 10, 275-283.

[20] Redline, R. W. Pappin A: Fetal thrombotic vasculopathy: The clinical significance of extensive avascular villi. Hum Pathol (1995). , 26, 80-85.

[21] Sander CM: Update: Etiologydiagnosis and management of hemorrhagic endovasculitis of the placenta. Compr Ther (1991). , 17, 16-19.

[22] Salafia, C. M, Pezzullo, J. C, Minior, V. K, et al. Placental pathology of absent and reversed end-diastolic flow in growth-restricted fetuses. Obstet Gynecol (1997). , 90, 830-836.

[23] Altemani, A. M. Sarian MZ: Hemorrhagic endovasculitis of the placenta: A clinicalpathological study in Brazil. J Perinat Med (1995). , 23, 359-363.

[24] Benirschke, K. Kaufman P: The Pathology of the Human Placenta (ed 3). New York, NY, Springer-Verlag, (1995). , 357-366.

[25] Fox H: Pathology of the Placenta (ed 2)Major Problems in Pathology, London, United Kingdom, Saunders, (1997). , 7, 118-122.

[26] Redline, R. W, Wilson-costello, D, Borawski, E, et al. Placental lesions associated with neurologic impairment and cerebral palsy in very low-birth-weight infants. Arch Pathol Lab Med (1998). , 122, 1091-1098.

[27] Rai, R, Regan, L, Hadley, E, et al. Second-trimester pregnancy loss is associated with activated C resistance. Br J Haematol (1996). , 92, 489-490.

[28] Rai RlRegan L, Chitolie A, et al: Placental thrombosis and second trimester miscarriage in association with activated protein C resistance. Br J Obstet Gynaecol (1996). , $103,842-844$. 
[29] Dizon-townson, D. S, Meline, L, Nelson, L. M, Varner, M, \& Ward, K. Fetal carriers of the factor $\mathrm{V}$ Leiden mutation are prone to miscarriage and placental infarction. Am J Obstet Gynecol (1997). , 177, 402-405.

[30] Von Kries, R, Junker, R, Oberle, D, Kosch, A, \& Nowak-göttl, U. Foetal growth restriction in children with prothrombotic risk factors. Thromb Haemost (2001). , 86, 1012-1016.

[31] Reverdiau-moalic, P, Delahousse, B, Body, G, et al. Evolution of blood coagulation activators and inhibitors in the healthy human fetus. Blood (1996). , 88, 900-906.

[32] Kahn, S. R, Platt, R, Mcnamara, H, Rozen, R, \& Chen, M. F. Genest J Jr, Goulet L, Lydon J, Seguin L, Dassa C, Masse A, Asselin G, Benjamin A, Miner L, Ghanem A, Kramer MS ((2009). Inherited thrombophilia and preeclampsia within a multicenter cohort: the Montreal Preeclampsia Study. Am J Obstet Gynecol 2009; 200:151.e, 1-9.

[33] Papageorghiou, A. T, \& Yu, C. K. Nicolaides KH: The role of uterine artery Doppler in predicting adverse pregnancy outcome. Best Pract Res Clin Obstet Gynaecol (2004). , 18, 383-396.

[34] Nicolaides, K. H, Bindra, R, Turan, O. M, et al. A novel approach to first-trimester screening for early pre-eclampsia combining serum and Doppler ultrasound. Ultrasound Obstet Gynecol (2006). , 13.

[35] Erden, O, Imir, A, Guvenal, T, Muslehiddinoglu, A, Arici, S, Cetin, M, \& Cetin, A. Investigation of the effects of heparin and low molecular- weight heparin on E-cadherin and laminin expression in rat pregnancy by immunohistochemistry. Hum Reprod (2006). , 21, 3014-3018.

[36] Grandone, E, Colaizzo, D, Martinelli, P, \& Paladini, D. di Minno G, Margaglione M. Adverse outcome in women with thrombophilia and bilateral uterine artery notches. Fertil Steril (2006). , 86, 726-727.

[37] Salomon, O, Seligsohn, U, Steinberg, D. M, Zalel, Y, Lerner, A, Rosenberg, N, Pshithizki, M, et al. The common prothrombotic factors in nulliparous women do not compromise blood flow in the fetomaternal circulation and are not associated with preeclampsia or intrauterine growth restriction. Am J Obstet Gynecol (2004). , 191, 2002-2009.

[38] Lazzarin, N, Vaquero, E, Exacoustos, C, Romanini, E, Amadio, A, \& Arduini, D. Midluteal phase Doppler assessment of uterine artery blood flow in nonpregnant women having a history of recurrent spontaneous abortions: correlation to different etiologies. Fertil Steril (2007). , 87, 1383-1387.

[39] Lindqvist, P. G, Procházka, M, Laurini, R, \& Maršál, K. Umbilical artery Doppler in relation to placental pathology and FV Leiden in pregnant women and their offspring. J Matern Fetal Neonatal Med. (2013). under press] 
[40] Cok, T, Tarim, E, \& Iskender, C. Comparison of uterine artery Doppler in pregnant women with thrombophilia treated by LMWHs and without thrombophilia. Arch Gynecol Obstet. (2012). , 286, 575-579.

[41] Magriples, U, Ozcan, T, Karne, A, \& Copel, J. A. The effect of anticoagulation on antenatal ultrasound findings in pregnant women with thrombophilia. J Matern Fetal Neonatal Med. (2006). , 19, 27-30.

[42] Alkazaleh, F, Viero, S, Simchen, M, Walker, M, Smith, G, Laskin, C, Windrim, R, \& Kingdom, J. Ultrasound diagnosis of severe thrombotic placental damage in the second trimester: an observational study. Ultrasound Obstet Gynecol. (2004). , 23, 472-6. 
